\title{
自适应多点定标非均匀性校正算法
}

\author{
黄 宇，张宝辉，吴杰，陈莹妍，吉 莉，吴旭东，于世孔
}

(昆明物理研究所, 云南 昆明 650223)

\begin{abstract}
摘要: 多点定标法校正精度较高, 但需要选择合理的标定点才能达到理论的校正效果。本文提出了一 种用于选择多点法标定点的算法。算法将残差作为选择标定点的判定条件, 自适应地在焦平面响应曲 线上确定标定点。探测器采集的原始图像经本文算法校正后图像的响应率不均匀性为 $0.31 \%$, 与传统 的均匀分段多点法相比减少了约 31\%, 多点法的校正精度得到了明显提高。
\end{abstract}

关键词: 自适应; 红外图像; 非均匀性校正

中图分类号：TP391 文献标识码：A 文章编号：1001-8891(2020)07-0637-07

\section{Adaptive Multipoint Calibration Non-uniformity Correction Algorithm}

\author{
HUANG Yu, ZHANG Baohui, WU Jie, CHEN Yingyan, JI Li, WU Xudong, YU Shikong \\ (Kunming Institute of Physics, Kunming 650223, China)
}

\begin{abstract}
The multipoint calibration method provides high calibration accuracy. However, a reasonable number of calibration points are required in order to achieve the theoretical correction effect. This work proposes an algorithm for selecting multipoint legal punctuation. The algorithm uses the residual as the criterion for selecting calibration points and adaptively determines the calibration points on the focal plane response curve. The non-uniformity of the response rate of the original image collected by the detector and corrected by the proposed algorithm is $0.31 \%$, which is about $31 \%$ less than that of the traditional uniform segmented multipoint method. In addition, the correction accuracy of the multipoint method is significantly improved.
\end{abstract}

Key words: adaptive, infrared image, non-uniformity correction

\section{0 引言}

凝视型红外焦平面阵列探测器目前广泛用于军 事、民用领域，但因制作工艺和材料引起的非均匀性 仍严重影响成像效果 ${ }^{[1]}$, 需要通过非均匀校正操作才 能达到实际应用要求 ${ }^{[2-3]}$ 。目前红外焦平面阵列 (Infrared focal plane array, IRFPA) 非均匀性校正的 方法主要有两大类: 一类基于场景, 利用实际场景信 息实现非均匀性校正 ${ }^{[4]}$; 另一类参考标准辐射源定标, 实现非均匀性校正 ${ }^{[5]}$ 。基于场景的方法具有自适应性, 可以减轻或消除漂移的影响, 但因收玫参数难以实现 快速稳定收玫, 而且算法的运算量大, 受到当前硬件 条件的制约, 很难实现实时校正 ${ }^{[6]}$ 。基于定标的方法 具有算法简单、计算量小, 可以实现实时校正的优点, 被广泛用于工程中 ${ }^{[7]}$ 。本文在基本的多点定标校正法 基础上提出一种新的温度区间划分方法, 提高定标校
正的精度, 扩大动态范围, 实现自适应多点定标校正。

\section{1 基本定标算法}

参考标准辐射源定标的校正方法常用的有单点、 两点、多点定标校正方法 ${ }^{[8]}$ 。

\section{1 单点定标校正法}

单点定标法是将所有像元在某一热辐射条件下 的输出信号校正为同一值。实现方法是将输出信号与 一个偏置参数 $\beta$ 相加来实现, 即图 1 展示的过程, 校 正后输出响应:

$$
\hat{V}_{(i, j)}=\beta_{(i, j)}+V_{(i, j)}
$$

式中: $\hat{V}_{(i, j)} 、 \beta(i, j) 、 V(i, j)$ 分别是坐标为 $(i, j)$ 的像元校 正后输出信号以及像元对应的偏置参数和输入信号。

由于单点校正法只能在标定点处将输出信号校 正一致, 所以单点法校正精度会随着偏离标定点的程 度增加迅速变差 ${ }^{[3]}$ 。 


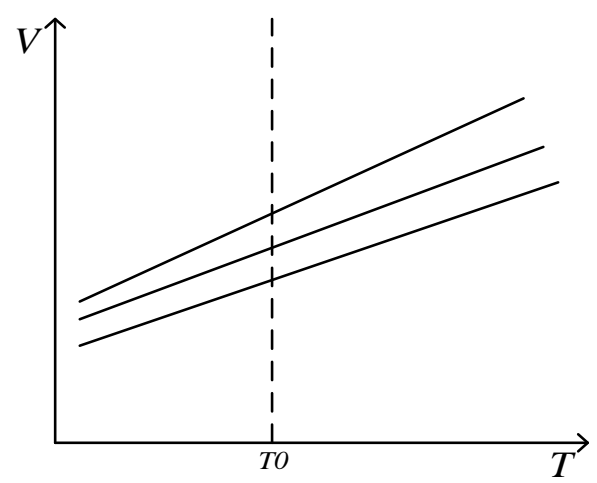

图 1 单点校正法

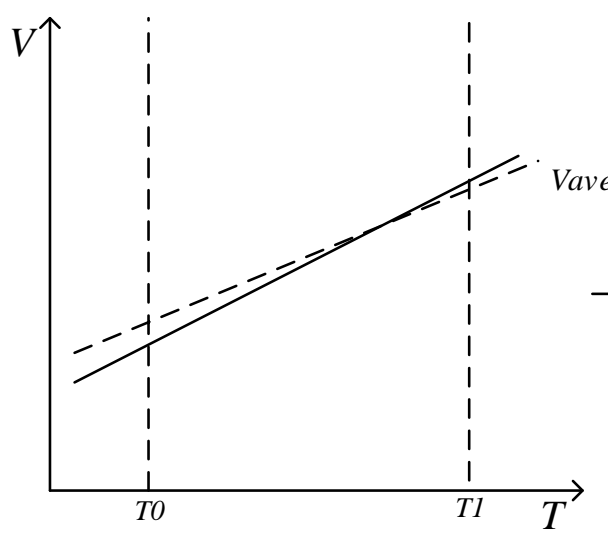

图 2 两点校正法

\section{2 两点定标校正法}

两点定标非均匀性校正算法是常用的校正算法 之一, 两点校正法校正过程如图 2 所示, 实现方法是: 将 $T_{\mathrm{L}} 、 T_{\mathrm{H}}$ 两个温度时的黑体辐射条件作为标定点, 用探测器得到辐射源为对应温度黑体时的均匀红外 辐射图像, 计算得到黑体温度为 $T_{\mathrm{L}} 、 T_{\mathrm{H}}$ 时的像元响 应 $V_{\mathrm{L}(i, j)} 、 V_{\mathrm{H}(i, j)}$ 和像元平均响应 $\overline{\mathrm{L}}_{\mathrm{L}}$ 和 $\bar{V}_{\mathrm{H}}$, 通过公式(1) 得到各像元的增益 $a_{(i, j)}$ 和偏置 $b_{(i, j)}$ :

$$
\begin{aligned}
& a_{(i, j)}=\frac{\bar{V}_{\mathrm{H}}-\bar{V}_{\mathrm{L}}}{V_{\mathrm{H}(i, j)}-V_{\mathrm{L}(i, j)}} \\
& b_{(i, j)}=\frac{V_{\mathrm{H}(i, j)} \bar{V}_{\mathrm{L}}-V_{\mathrm{L}(i, j)} \bar{V}_{\mathrm{H}}}{V_{\mathrm{H}(i, j)}-V_{\mathrm{L}(i, j)}}
\end{aligned}
$$

校正后的像元输出响应 $\hat{V}_{(i, j)}=a_{(i, j)} V_{(i, j)}+b_{(i, j)}$ 。

两点定标校正法的提出建立在两个假设上: (1)像 元的响应在两个标定点之间满足线性模型; (2)像元对 相同强度的辐射输出响应基本不变 ${ }^{[10]}$ 。然而红外焦平 面的实际响应曲线并不完全满足线性模型, 只在一个 较小的温度区间内近似满足线性模型, 两点校正法只 能保证输入响应在两标定点之间时的校正精度, 算法

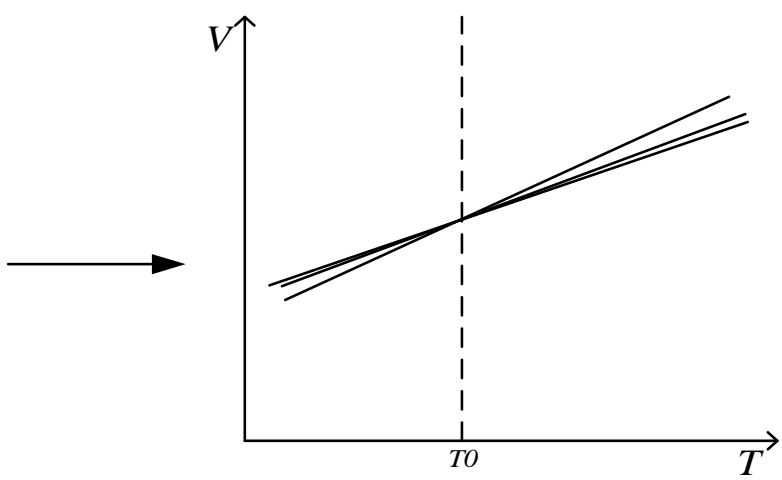

Fig.1 Single point correction

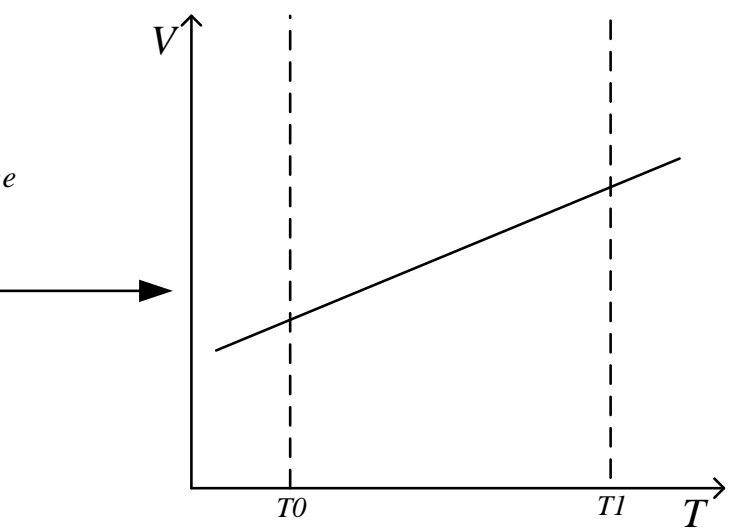

Fig.2 Two-point correction

模型和响应曲线的非线性造成的残差会随着温度 $T$ 与 标定温度间的距离增大而增大，因此两点校正法定标 得到的参数 $a_{(i, j)} 、 b_{(i, j)}$ 有效作用范围较小, 难以满足 大动态温度范围校正的需要。

\section{3 多点定标校正法}

为了克服两点定标校正算法参数的有效温度范 围小，及建立的模型会因响应曲线的非线性引入残差 的缺点, 在两点法的基础上提出了多点定标校正法, 多点校正法与两点法简单的使用线性模型来代替实 际响应曲线不同，多点法承认响应曲线具有非线性， 用折线模型来逼近实际的响应曲线，折线的每段都用 两点定标法计算对应的 $a_{n(i, j)} 、 b_{n(i, j)}, n$ 为折线段的序 号。定标后储存一系列偏置参数 $a_{n(i, j)}$ 、增益参数 $b_{n(i,}$ ${ }_{j}^{j}$, 以及线段端点对应的校正前像元响应用于校正操 作。在校正操作时, 先根据像元的校正前响应判断所在 区间, 找到区间对应的 $a_{n(i, j)} 、 b_{n(i, j)}$, 计算出校正后的 像元输出响应 $\hat{V}_{(i, j)}$ 。

从多点定标校正算法计算过程中可以发现, 标定 点决定多点定标校正法的校正精度。文献[2]分析了标 定点对非均匀性校正精度的影响, 得出了标定点数越 多校正精度越高; 选取的标定点温度不同, 校正精度 
也不同的结论 ${ }^{[2]}$ 。可见, 选择合理的标定点是提高多 点定标校正法的算法性能及校正精度的关键。

\section{2 自适应多点定标校正算法}

每一台焦平面探测器的响应曲线都不完全相同, 存在一定的差异, 因此在对不同的探测器进行定标时 需要选择不同的标定点, 这样才能得到较好的校正效 果。人工选择标定点效率低且无法保证获得的标定点 的校正效果, 因此自动地获得合理的标定点的方法成 为了多点定标校正法使用中的重要部分。

如图 3 所示曲线 $A C D B$ 为一条响应曲线, $A B$ 为 两点校正的计算结果, 显然直线 $A B$ 与曲线 $A C D B$ 间 的残差随距离增大而增大, 越接近区间 $\left(T_{A}, T_{B}\right)$ 的中点 残差越大。但曲线不同残差值最大的位置也不同。C 为区间 $\left(T_{A}, T_{B}\right)$ 的中点, 折线 $A C B$ 为将区间 $\left(T_{A}, T_{B}\right)$ 均 分两段后得到的多点法的折线模型, 可见看到折线 $A C B$ 在 $A C$ 段残差较小, 但在 $B C$ 段残差很大, 这样 无法在区间 $\left(T_{A}, T_{B}\right)$ 中始终保持较好的校正效果, 所以 需要用其他方法来确定分段点的位置。

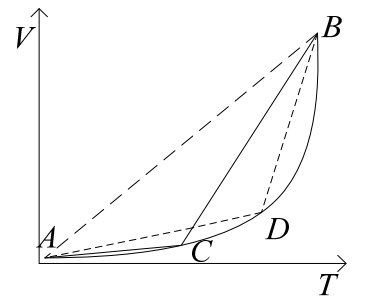

图 3 多点校正原理

Fig.3 Multi-point correction principle

为了使多点校正法在工作区间内始终保持较好 的校正效果, 就要让折线始终尽可能地逼近响应曲 线, 即使残差的平方和达到最小。对于多点校正的折 线模型来说, 为使残差的平方和尽可能小, 可以用残 差平方最大的点作为折线模型的端点的方法, 来减小 残差的平方和。

设点 $D$ 为残差最大的点, 以该点为端点, 那么多 点校正的模型为折线 $A D B$, 从图中可以发现虽然在 $\left(T_{A}, T_{C}\right)$ 区间上均分法的残差比最大残差处分段法的 残差小, 更加贴合响应曲线, 但最大残差处分段法能 在全区间 $\left(T_{A}, T_{B}\right)$ 上保持较小的残差, 逼近曲线 $A C D B$, 达到较好的校正效果。

这里, 提出一个自适应的用于获得合理的多点定 标校正法分段区间的方法，使用残差作为区间端点判 定依据。

首先, 设响应曲线的起点为 $P_{1}$, 终点为 $P_{n}$, 那 么可以得到直线 $P_{1} P_{n}$ :

$$
V_{\text {line }}^{0}=\frac{V_{n}-V_{1}}{T_{n}-T_{1}} T+\frac{V_{1} T_{n}-V_{n} T_{1}}{T_{n}-T_{1}}
$$

式中: $V_{1}$ 和 $V_{n}$ 分别为起点、终点处响应曲线的值; $V_{\text {line }}$ 为计算得到的响应。

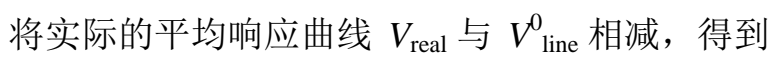
各点残差 $\Delta^{0}(i)$ :

$$
\Delta^{0}(i)=\left|V_{\text {real }}(i)-V_{\text {line }}^{0}(i)\right|
$$

设 $T=T_{m 1}$ 时残差最大, 即:

$$
\max \left\{\Delta^{0}(i)\right\}=\Delta^{0}(m 1)(i=1,2, \cdots, n)
$$

令 $P_{m 1}$ 成为新的区间端点, 那么由 $P_{1} P_{m 1} P_{n}$ 可以 得到新的折线 $V_{\text {line }}^{1}$ :

$$
V_{\text {line }}^{1}=\left\{\begin{array}{l}
\frac{V_{m 1}-V_{1}}{T_{m 1}-T_{1}} T+\frac{V_{1} T_{m 1}-V_{m 1} T_{1}}{T_{m 1}-T_{1}}(1 \leq T \leq m 1) \\
\frac{V_{n}-V_{m 1}}{T_{n}-T_{m 1}} T+\frac{V_{m 1} T_{n}-V_{n} T_{m 1}}{T_{n}-T_{m 1}}(m 1<T \leq n)
\end{array}\right.
$$

与式(5)同理, 可以得到各点处新的残差 $\Delta^{1}(i)$ 。设 $T=T_{m 2}$ 时残差 $\Delta^{1}$ 最大, 且 $T_{m 2} \in\left(T_{m 1}, T_{n}\right)$, 那么新的折 线 $V_{\text {line }}^{2}$ :

$$
V_{\text {line }}^{2}=\left\{\begin{array}{l}
\frac{V_{m 1}-V_{1}}{T_{m 1}-T_{1}} T+\frac{V_{1} T_{m 1}-V_{m 1} T_{1}}{T_{m 1}-T_{1}}(1 \leq T \leq m 1) \\
\frac{V_{m 2}-V_{m 1}}{T_{m 2}-T_{m 1}} T+\frac{V_{m 1} T_{m 2}-V_{m 2} T_{m 1}}{T_{m 2}-T_{m 1}}(m 1<T \leq m 2) \\
\frac{V_{n}-V_{m 2}}{T_{n}-T_{m 2}} T+\frac{V_{m 2} T_{n}-V_{n} T_{m 2}}{T_{n}-T_{m 2}}(m 2<T \leq n)
\end{array}\right.
$$

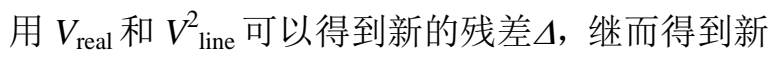
的区间端点。重复迭代上述过程就可获得足够多的区 间端点。算法的流程图如图 4 所示。

自适应多点校正法使用残差作为分段端点的判 定依据, 与传统的均分多点校正法相比, 能够更好地 适应不同探测器的不同响应曲线, 使用相同的分段数 目更好地逼近响应曲线, 在全局上保持了较小的残 差, 从而保证了校正精度。

\section{3 性能测试}

为了评估自适应多点校正法的校正效果，将其与 两点校正法、均分多点校正法的校正效果进行比较, 做了性能测试。性能评价指标选择 ${ }^{[9]}$ 中响应率不均匀 性（responsivity non-uniformity, UR）式(7)计算:

$$
\mathrm{UR}=\frac{1}{\bar{R}} \sqrt{\frac{1}{M \times N-(d+h)} \sum_{i=1}^{M} \sum_{j=1}^{N}[R(i, j)-\bar{R}]^{2}} \times 100 \%(7)
$$


式中: $\bar{R}$ 为平均响应率; $M$ 为像元总行数; $N$ 为像元 总列数; $d$ 和 $h$ 分别为死像元和过热像元; $R(i, j)$ 为像 元响应率 ${ }^{[9]}$ 。

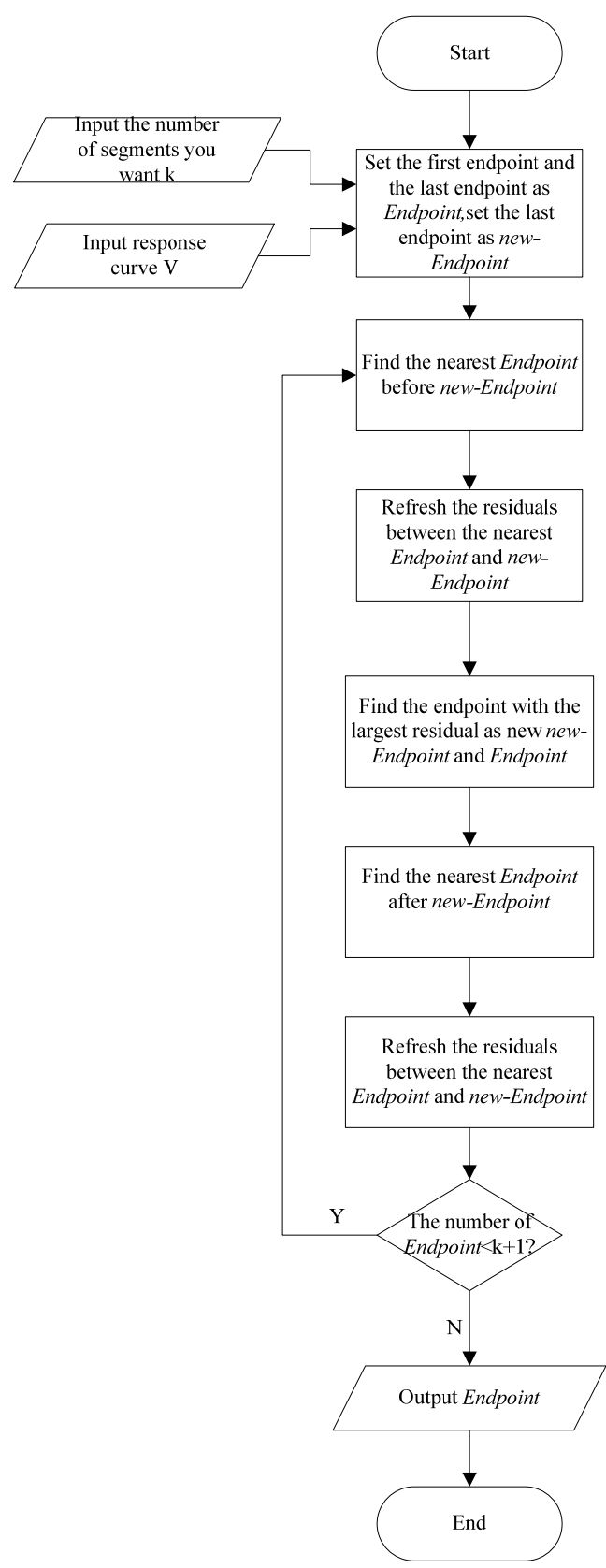

图 4 算法流程图 Fig.4 Algorithm flowchart 死像元和过热像元同样使用文献[9]中的定义即:

$$
\begin{aligned}
& R(i, j)<\frac{1}{2} \bar{R} \\
& V_{N}(i, j)>2 \bar{V}_{N}
\end{aligned}
$$

测试数据是使用图 5 所示国产某型 $640 \times 512$ 制 冷型红外焦平面探测器采集面源黑体 (图 6) 得到的 均匀辐射黑体图像, 黑体温度变化范围为 $278 \mathrm{~K} \sim 323$ $\mathrm{K}$, 采样温度间隔为 $1 \mathrm{~K}$, 同一温度采集 60 帧图像, 最终得到 46 组图像。为避免随机性噪声干扰, 定标 时各温度点的图像为 60 帧图像的空间平均。

首先进行盲元判定, 剔除盲元对定标的影响。判 定时选取的黑体温度分别为 $308 \mathrm{~K} 、 293 \mathrm{~K}$, 得到的盲 元总数为 598 个, 其中死像元 347 个, 过热像元 251 个, 盲元占总像元的比例为 $0.18 \%$ 。

标定操作使用平均响应 $\bar{V}$-黑体温度 $T$ 曲线, 区 间划分结果如图 7 所示。

虚线为两种区间划分方式各自的折线模型, 实线 为平均响应曲线, 空心圆点为定标点。两种算法的分 段结果在图 7 中仅有细微的差别, 肉眼难以分辨, 因 此将两折线分别与平均响应曲线相减得到各自的残 差, 绘制图 8 便于观察区别。

在全部 46 个温度点, 除两侧端点外剩余 44 个点 中, 有 18 个点（包含平均分段残差的 3 个区间端点） 处最大残差分段法的残差大于平均分段法的残差; 有 26 个点 (包含最大残差分段残差的 3 个区间端点) 处 最大残差分段法的残差小于平均分段法的残差。计算 两种方法的残差平方和, 平均分段法的残差平方和为 35097 , 最大残差分段法的残差平方和为 20131, 明显 小于平均分段法的残差平方和。使用最大残差法划分 区间得到的多点法校正模型与响应曲线拟合得更好, 且残差最大值比平均分段法的残差最大值要小, 非均 匀校正的效果也更好。

分别使用两点法、均匀分段多点法、自适应多点 法对采集的数据定标校正, 计算校正后结果的响应率 不均匀性（UR），结果见表 1 。

UR 的计算结果与理论分析得到结论相同，使用 折线模型的多点法对非均匀性的校正效果比两点法 的校正效果要好。自适应多点法的校正效果比均匀分 段多点法的效果要好, 而且效果提升明显, UR 减少 了约 $31 \%$ 。

任选一帧黑体图像, 使用两点法、均匀分段多点 法、自适应多点法进行非均匀性校正, 图 9 为校正前 的原始图像, 3 种不同方法的校正结果见图 10、图 11、 图 12。 


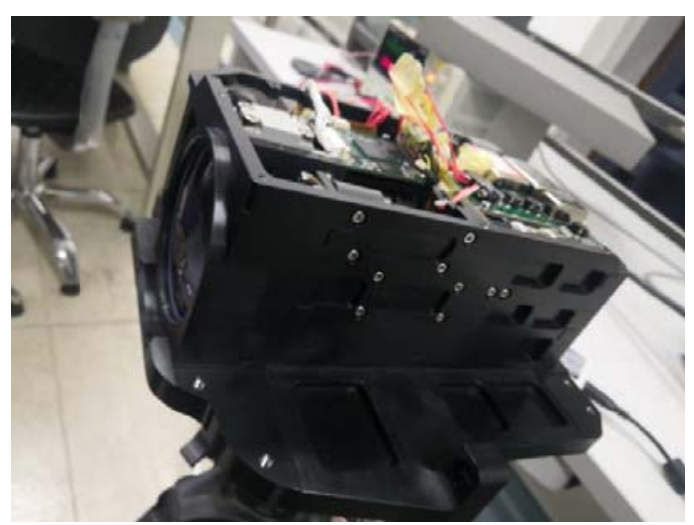

图 5 国产某型 $640 \times 512$ 制冷型红外焦平面探测器

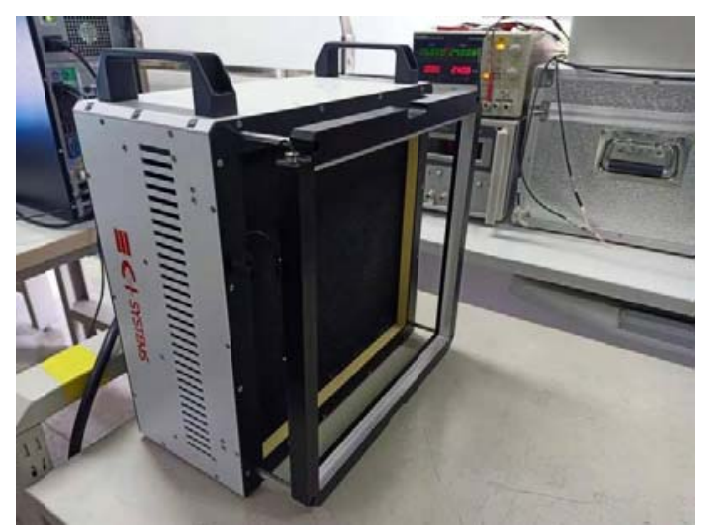

图 6 黑体

Fig.5 A domestic $640 \times 512$ refrigerated infrared focal plane detector

Fig.6 Black body

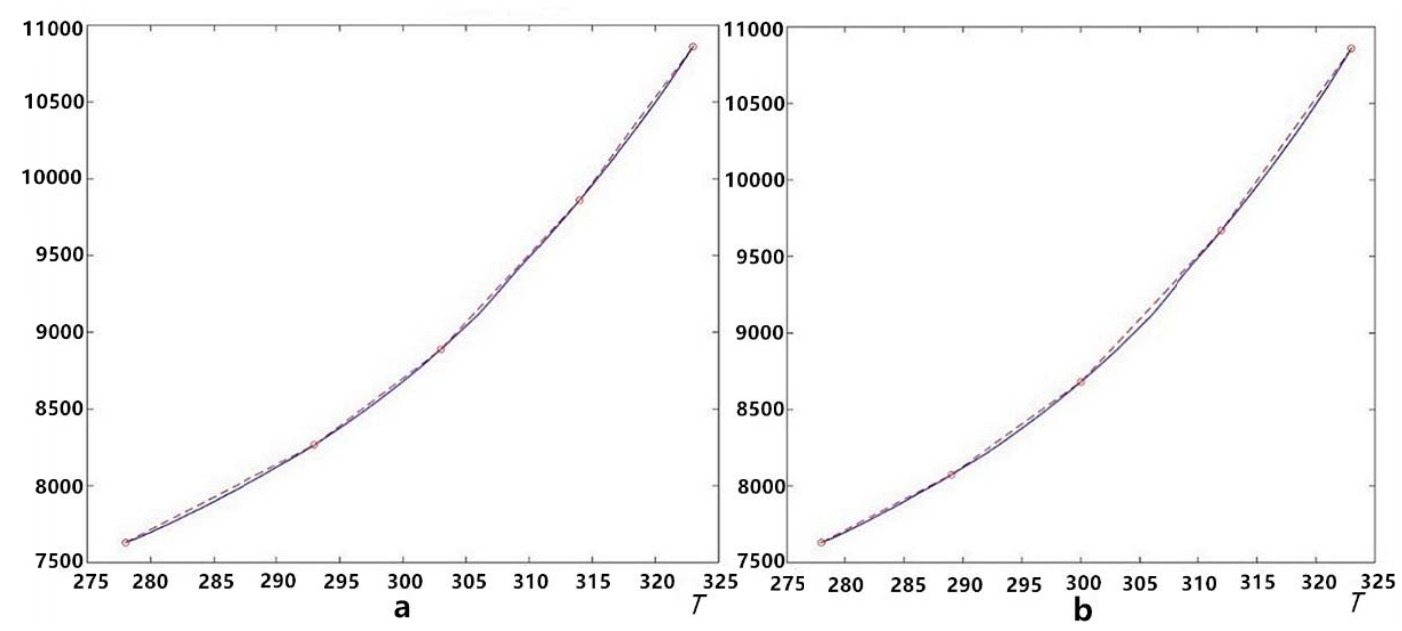

图 7 分段结果, (a)文中提出的算法(b)平均分段多点法

Fig.7 Segmentation results, (a)Proposed algorithm, (b) mean segmented multi-point correction algorithm

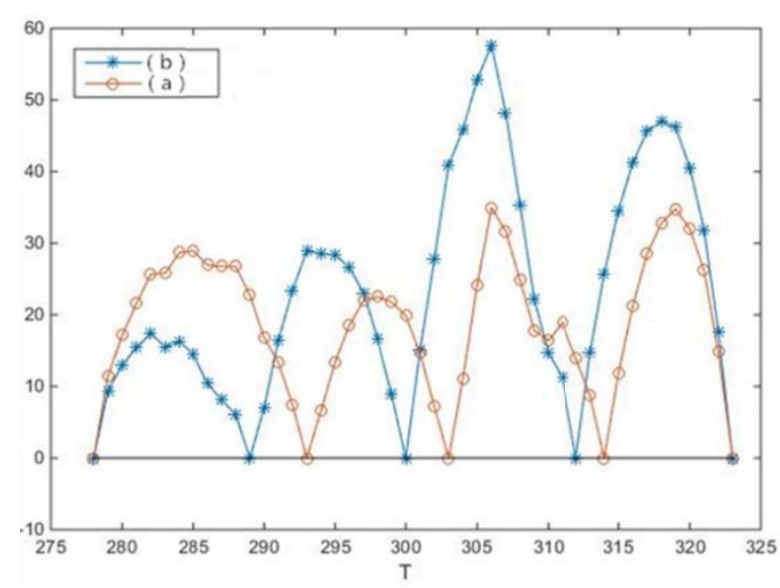

图 8 图 7 中两种算法的残差

Fig.8 Residuals of the two algorithms in Fig.7
表 1 四种算法 UR 比较

Table1 UR Comparison of four algorithms

\begin{tabular}{lllll}
\hline $\begin{array}{l}\text { Correction } \\
\text { algorithm }\end{array}$ & $\begin{array}{l}\text { Original } \\
\text { image }\end{array}$ & $\begin{array}{l}\text { Two-point } \\
\text { algorithm }\end{array}$ & $\begin{array}{l}\text { Mean segmented } \\
\text { multi-point }\end{array}$ & Proposed \\
\hline $\mathrm{UR}$ & $6.81 \%$ & $0.59 \%$ & $0.45 \%$ & $0.31 \%$ \\
\hline \multicolumn{4}{c}{ 从人眼视觉角度来看，图 10 两点法的校正结果 }
\end{tabular}
有明显坚条纹残留, 且左右两侧明暗差别明显; 图 11 平均分段多点法的校正结果中坚条纹不明显, 但仍有 完整的坚条纹存在, 右侧阴影残留与两点法校正结果 相比有改善，但仍然明显; 图 12 自适应多点法校正 结果中无完整坚条纹存在, 也无明显明暗色差, 右下 角处阴影几乎完全消失。从校正结果来看, 校正效果 为 3 种方法中最好。在图 13 中两种多点法实际场景 校正效果的对比进一步证明自适应多点法校正效果 优于平均分段多点法。 


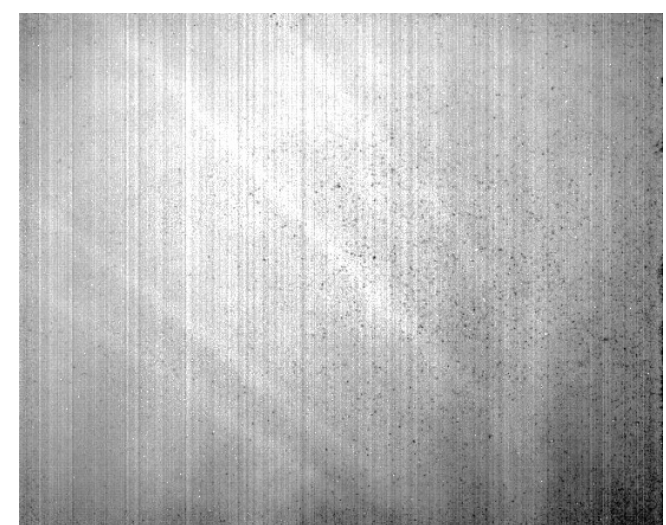

图 9 原始图像 Fig.9 Original image

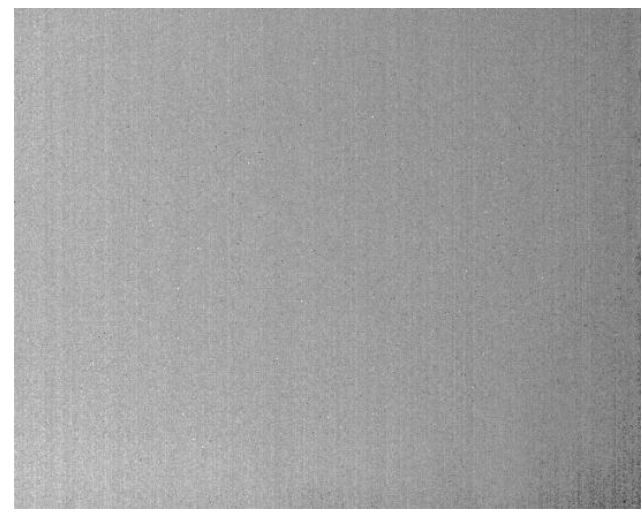

图 11 平均分段多点法校正结果

Fig.11 Mean segmented multi-point correct result
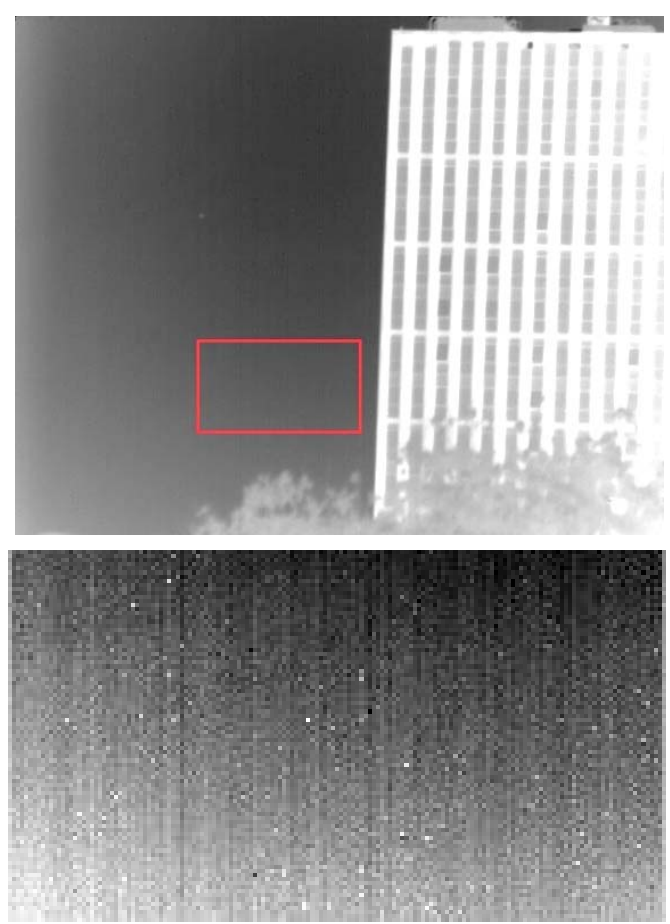

(a) 平均分段多点法

(a) Mean segmented multi-point correct

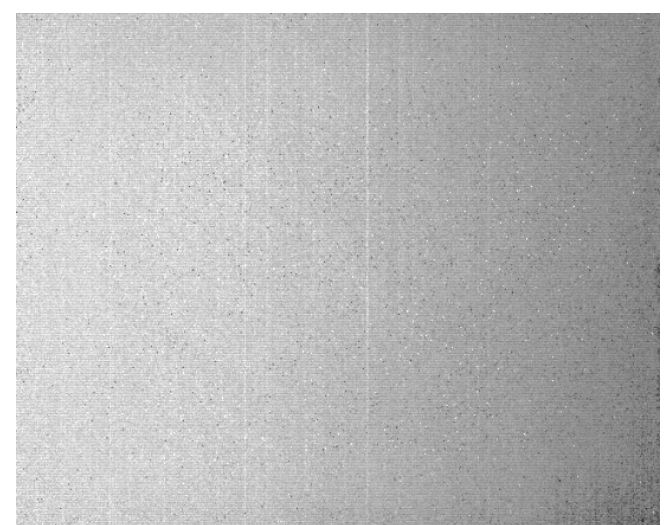

图 10 两点法校正结果 Fig.10 Two-point correct result

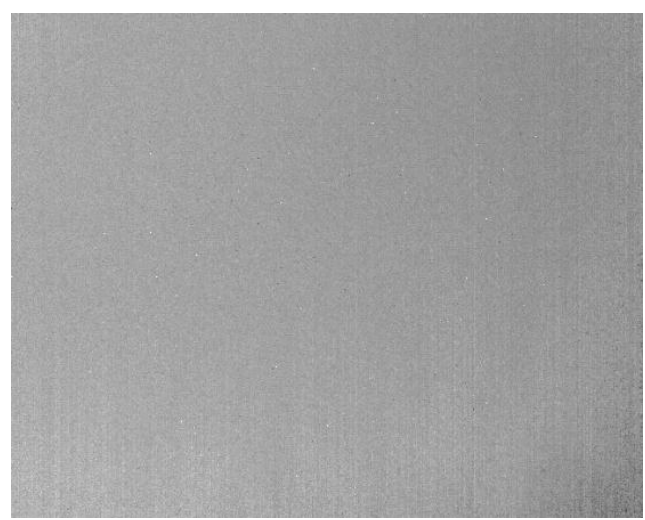

图 12 自适应多点法校正结果

Fig.12 Adaptively segmented multi-point correct result
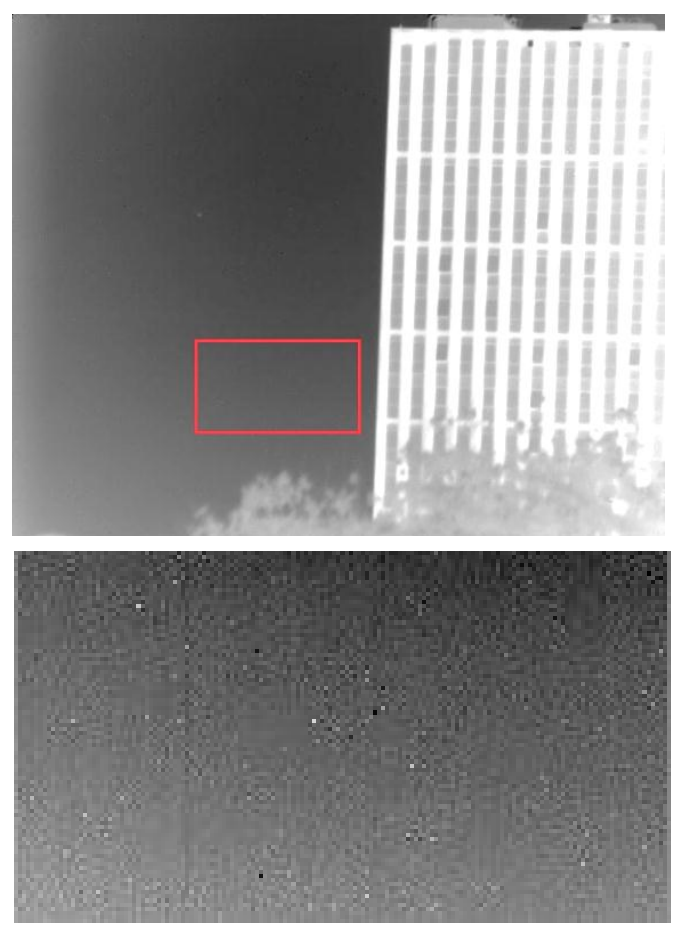

(b) 自适应多点法

(b) adaptively segmented multi-point correct

图 13 两种方法对场景校正结果及局部放大图(a) (b)

Fig.13 Two methods for scene correction results and local zoom 


\section{4 结论}

多点定标校正法是定标校正法的一种, 算法模型 接近实际响应曲线。为实现算法理论上的校正效果, 本文提出了一个方法用来自适应地确定合理的标定 点。在理论论证方法可行后, 使用了 $640 \times 512$ 制冷 型红外探测器采集数据用来对本文方法进行实验分 析。实验结果表明, 使用本文方法的自适应分段多点 法的图像非均匀性校正效果与基本的均匀分段多点 法校正效果相比有明显提升, 响应率不均匀性减少了 约 31\%。校正后输出的红外图像质量改善明显, 对后 续图像增强等操作有重要意义。

\section{参考文献:}

[1] 刘源, 李庆, 梁艳菊. 基于 FPGA 的红外目标自动检测系统 [J]. 红外技 术, 2019, 41(6): 521-526.

LIU Yuan, LI Qing, LIANG Yanju. Implementation of Infrared Target Detection System Based on FPGA[J]. Infrared Technology, 2019, 41(6): 521-526.

[2] 姚琴芬, 顾国华. 红外焦平面阵列非均匀性校正精度的研究[J]. 红外, 2012, 33(8): 22-26.

YAO Qinfen, GU Guohua. Study of nonuniformity correction precision of IRFPA[J]. Infrared, 2012, 33(8): 22-26.

[3] 雷晓杰, 顾国华, 隋修宝, 等. 红外双边滤波时域高通非均匀性校正 [J]. 激光与红外, 2012, 42(7): 831-836.

LEI Xiaojie, GU Guohua, SUI Xiubao, et al. Improved temporal high-pass filter nonuniformity correction based on bilateral filter[J]. Laser Infrared, 2012, 42(7): 831-836.

[4] 李谦, 杨波, 粟宇路, 等. 基于神经网络的红外焦平面光学非均匀性 校正改进算法[J]. 红外技术, 2019, 41(3): 251-255.
LI Qian, YANG Bo, SU Yulu, et al. An Improved Algorithm for IRFPA Optical Nonuniformity Correction Based on Neural Networks[J]. Infrared Technology, 2019, 41(3): 251-255.

[5] 郁品一. 多点定标非均匀性校正方法的综合应用 [J]. 红外与激光工程, 2008, 37(S2): 615-618.

YU Pinyi. Application of multi-point nonuniformity correction method[J]. Infrared and Laser Engineering, 2008, 37(S2): 615-618.

[6] 张宝辉, 姚立斌, 张巍伟, 等. 红外图像校正与增强技术研究现状 [J]. 红外技术, 2017, 39(6): 481-488.

ZHANG Baohui, YAO Libin, ZHANG Weiwei, et al. The current research status of infrared image correction and enhancement[J]. Infrared Technology, 2017, 39(6): 481-488.

[7] 吕雷, 张学峰. 基于 FPGA 的红外图像实时非均匀性校正 $[\mathrm{J}]$. 激光与 红外, 2011, 41(6): 641-643.

LYU Lei, ZHANG Xuefeng. Real-time infrared image nonuniformity correction base on FPGA[J]. Laser Infrared, 2011, 41(6): 641-643.

[8] 王琨. 红外成像系统非均匀校正技术研究[D]. 成都: 电子科技大学, 2017.

WANG Kun. Research on marching-on in-time scheme and the fast algorithm of time domain integral equation[D]. Chengdu: University of Electronic Science and Technology of China, 2017.

[9] 中华人民共和国工业和信息化部. 红外焦平面阵列参数测试方法: GB/T 17444-2013[S]. 北京: 中国标准出版社, 2014.

Ministry of Industry and Information Technology of the People's Republic of China. Measuring methods for parameters of infrared focal plane arrays :GB/T 17444-2013[S]. Beijing: Standards Press of China, 2014.

[10] 颜益. 红外焦平面非均匀校正及图像增强研究 [D]. 厦门: 厦门大学, 2009.

YAN Yi. Study on nonuniformity correction algorithm and image enhancing algorithm on IRFPA[D]. Xiamen: Xiamen University, 2009. 